\title{
Which prosodic features contribute to the recognition of dramatic attitudes?
}

\author{
Adela Barbulescu ${ }^{\mathrm{a}, *}$, Rémi Ronfard ${ }^{\mathrm{a}}$, Gérard Bailly ${ }^{\mathrm{b}}$ \\ a University Grenoble Alpes, Inria, LJK, France \\ ${ }^{\mathrm{b}}$ GIPSA-lab, CNRS \& University Grenoble Alpes, Grenoble, France
}

\section{A R T I C L E I N F O}

\section{Article history:}

Received 15 January 2017

Revised 16 July 2017

Accepted 28 July 2017

Available online 1 August 2017

\section{Keywords:}

Audiovisual expressive speech

Affective database

Dramatic attitudes

Perceptual correlates

\begin{abstract}
A B S T R A C T
In this work we explore the capability of audiovisual prosodic features (such as fundamental frequency, head motion or facial expressions) to discriminate among different dramatic attitudes. We extract the audiovisual parameters from an acted corpus of attitudes and structure them as frame, syllable and sentence-level features. Using Linear Discriminant Analysis classifiers, we show that prosodic features present a higher discriminating rate at sentence-level. This finding is confirmed by the perceptual evaluation results of audio and/or visual stimuli obtained from the recorded attitudes.
\end{abstract}

\section{Introduction}

Attitudes refer to the expression of social affects and present acoustic and visual manifestations which are linked to conventions and cultural behaviors (Scherer, 1986). Thus, attitudes differ from basic emotional expressions, which may be seen as more spontaneous and universal expressions (Scherer et al., 1979; Scherer and Wallbott, 1994).

The study of audiovisual parameters which encode the paralinguistic content of speech plays an essential role in improving the recognition and synthesis of expressive audiovisual speech. To this goal, there has been a great amount of work on the analysis and modeling of features which are found to help in the discrimination between expressive styles. Audiovisual features such as voice quality (Monzo et al., 2007), acoustic prosodic features (F0, rhythm, energy) (Morlec et al., 2001; Iriondo et al., 2007; Mixdorff et al., 2015), head motion (Busso et al., 2007) and facial expressions (Davis et al., 2015), have proven to be efficient in discriminating between basic emotions, attitudes or speaker identity.

While recognition of emotion, psycho-physiological state or coverbal activities (drinking, eating, etc) is largely based on signalbased data mining and deep learning with features collected with

\footnotetext{
* Corresponding author.

E-mail addresses: adela.barbulescu@inria.fr, barbulescu.adela@gmail.com (A. Barbulescu),remi.ronfard@inria.fr (R. Ronfard), gerard.bailly@gipsa-lab.fr (G. Bailly).
}

a sliding window over multimodal frames, early studies on the expression of verbal attitudes have proposed that speakers use global prosodic patterns to convey an attitude (Fónagy etal., 1983; Bolinger, 1989). These patterns are supposed to be anchored on the discourse and its linguistic structure, rather than encoded independently on parallel multimodal features. We recently evidenced the relevance of such patterns in facial displays (Barbulescu et al., 2015).

The main aim of this work is to further explore the effectiveness of using audiovisual features at different structural levels to discriminate among expressive styles. We thus compare below the discrimination between attitudes at different structural levels (frame, syllable and sentence) and with different acoustic and visual features in order to evaluate the importance of the positioning of discriminant audiovisual events within the utterance. To that purpose, we performed a series of Linear Discriminant Analyses (LDA) on an expressive corpus of dramatic attitudes. In line with Iriondo etal. (2009) who used the results of a subjective test to refine an expressive dataset, we compare our best classification results with perceptual evaluation tests for the set of attitudes which are best discriminated.

The paper is structured as follows: Section 2 presents approaches in related studies, Section 3 presents our corpus of attitudes and the extraction of audiovisual features. Section 4 presents the experiments we carried out for automatic classification and Section 5 presents the perceptual evaluation and comparison techniques, followed by conclusions in Section 6. 
Table 1

Datasets for affect recognition systems.

\begin{tabular}{|c|c|c|c|c|c|c|}
\hline & \# subjects & \# samples & Data type & $\begin{array}{l}\text { Speech \& } \\
\text { \# sentences }\end{array}$ & Categories & Spontaneity \\
\hline BIWI Fanelli etal. (2010) & 14 & 1109 & 3D face $\&$ video & Yes - 40 & 11 affective labels & acted \\
\hline 4D Cardiff Vandeventer et al. (2015) & 4 & $\mathrm{~N} / \mathrm{A}$ & 3D face $\&$ video & Yes - N/A & 10 expressions & spontaneous \\
\hline $\begin{array}{l}\text { MPI facial expressions Kaulard et al. } \\
\text { (2012) }\end{array}$ & 6 & $\mathrm{~N} / \mathrm{A}$ & 3D face & $\mathrm{N} / \mathrm{A}$ & 55 expressions & acted \\
\hline Bosphorus Savran et al. (2008) & 105 & 4652 & 3D face (static) & $\mathrm{N} / \mathrm{A}$ & $\begin{array}{l}37 \text { expressions (action units, } \\
\text { basic emotions) }\end{array}$ & acted \\
\hline CK+ Lucey et al. (2010) & 123 & 593 & video & $\mathrm{N} / \mathrm{A}$ & $\begin{array}{l}23 \text { expressions (action units, } \\
\text { combinations) }\end{array}$ & posed and non-posed \\
\hline CAM3D Mahmoud etal. (2011) & 7 & 108 & 3D face $\&$ torso & Yes - N/A & 12 mental states & spontaneous \\
\hline Mind Reading Baron-Cohen (2003) & 6 & $\mathrm{~N} / \mathrm{A}$ & video & Yes - N/A & 412 emotions in 24 categories & acted \\
\hline IEMOCAP Busso et al. (2008) & 10 & $\mathrm{~N} / \mathrm{A}$ & 3D face $\&$ torso & $\mathrm{N} / \mathrm{A}$ & 8 emotions & acted and spontaneous \\
\hline
\end{tabular}

\section{Related work}

Although recent years have brought a substantial progress in the field of affective computing (Zeng etal., 2009), the development of emotion-modeling systems strongly depends on available affective corpora. As training and evaluation of algorithms require a great amount of data which is hard to collect, publicly available datasets represent a bottleneck for research in this field. Moreover, the majority of available datasets are limited to the six basic emotion categories proposed by Ekman and Friesen (1971) and include happiness, sadness, fear, anger, disgust, and/or surprise.

Databases containing affective data can be categorized under several criteria: data types used (2D or 3D visual data, speech), spontaneity (naturalistic, artificially induced or posed by professional actors or not), affective state categorization (emotion, attitudes etc). Audiovisual recording is obviously more expensive and time-consuming than audio-only recording. This is proven by the comparative amounts of publicly available audio and audiovisual datasets. For instance, the Interspeech Computational Paralinguistic Challenge ${ }^{1}$ provides audio data from a high diversity of speakers and different languages, such as (non-native) English, Spanish, and German.

A comprehensive overview of the existing audiovisual corpora can be obtained from Cowie etal. (2005); Zeng etal. (2009). Table 1 presents a set of expressive datasets which are most relevant to our work. The works listed in the table present publicly available data that are used in several research topics: analysis, affective recognition, expressive performance generation, audiovisual conversion etc. IEMOCAP Busso et al. (2008) and CAM3D Mahmoud etal. (2011) contain motion capture data of the face and upperbody posture from spontaneous performances. Large data variability is presented by Savran et al. (2008) and CK+ (Lucey et al., 2010) as they include more than 100 subjects posing over 20 expressions each, in the shape of action units and combinations. Another important work is the Mind Reading dataset (Baron-Cohen, 2003) which includes video recordings of 412 expressive states classified under 24 main categories. While they serve as valuable references for the expressive taxonomy, these datasets often do not contain audio data. To our knowledge, the only publicly available affective datasets that include 3D data and speech are the BIWI, CAM 3D and 4D Cardiff corpora. Although the expressive categories contained extend the set of basic emotions, only a few present conversational potential (thinking, confused, frustrated, confidence). Most importantly, the sentences used in the datasets do not present a high variability or a systematic variation of syllable lengths.

Using data collected from such databases, a large amount of studies have been conducted for the analysis of audiovisual prosody. Swerts and Krahmer (2010) present a detailed overview

\footnotetext{
1 http://compare.openaudio.eu/.
}

of studies carried out on audiovisual prosody. One aspect of this research relates directly to the communication functions that were traditionally attributed to auditory prosody: prominence, focus, phrasing. Analysis on head nods, eye blinks, eyebrows movement showed that these visual cues present a high influence on word prominence (Pelachaud etal., 1996; Granström etal., 2002; Cvejic et al., 2010). Other areas of study include emotion, attitude and modality. Busso etal. (2004) analyzed different combinations of acoustic information and facial expressions to classify a set of 4 basic emotions. The results showed that the acoustic and visual information are complementary. Improved results were also obtained by considering sentence-level features, especially for visual data. In Busso etal. (2007) authors performed statistical measures on an audiovisual database, revealing characteristic patterns in emotional head motion sequences. Ouni et al. (2016) analyze the acoustic (F0, energy, duration) and 3D visual data (facial expressions) captured by an actor performing 6 basic emotions. While no universal feature is found to discriminate between all the emotions, a few observations are noted: anger, joy, fear and surprise have similar speech rates, the facial movements are more important for joy, surprise and anger.

The expression of attitudes is highly dependent on the studied language. The following works focus on the study of attitudes and/or generation of prosody (intonation): Morlec et al. (2001) (French), Mac etal. (2012) (Vietnamese), De Moraes et al. (2010) (Brazilian Portuguese), Hnemann etal. (2015)(German). However, the datasets recorded for these studies are not publicly available and do not feature 3D data. De Moraes et al. (2010) conducted a perceptual analysis of audiovisual prosody for Brazilian Portuguese using video data recorded by two speakers. They studied 12 attitudes categorized as social (arrogance, authority, contempt, irritation, politeness and seduction), propositional (doubt, irony, incredulity, obviousness and surprise) and assertion (neutral). An attitude recognition test showed the following: the difference in perception between the two speakers for certain attitudes such as the different strategies developed for irony and seduction, different dominant modality such as one speaker is better recognized in audio while the other in video, better overall recognition rates for audio-video among all modalities, the propositional and social attitudes show different perceptual behaviors. Another work on perception of audiovisual attitudes is focused on the expression of 16 social and/or propositional attitudes in German. Hönemann et al. (2014) perform a set of attitude recognition tests. While the observations are valuable, these studies focus on the perceptual results of attitude recognition tests and do not carry out a complete analysis, including facial features and voice parameters.

To our knowledge, there are no extensive studies of the correlation between acoustic features and nonverbal gestures for the production of a large set of complex attitudes. Except for a few works related to modalities, such as interrogation (Srinivasan and 
Table 2

Presentation of chosen attitudes: category, subgroup and definition are shown as they appear in Mind Reading (Baron-Cohen, 2003).

\begin{tabular}{lllll}
\hline Category & Subgroup & Our labels & Abbr. & Definition \\
\hline Kind & Comforting & Comforting & CF & Making people feel less worried, unhappy or insecure \\
Fond & Liking & Tender & TE & Finding something appealing and pleasant; being fond of something \\
Romantic & Seductive & Seductive & SE & Physically attractive \\
Interested & Fascinated & Fascinated & FA & Very curious about and interested in something that you find impressive \\
Thinking & Thoughtful & Thinking & TH & Thinking deeply or seriously about something \\
Disbelieving & Incredulous & Doubtful & DO & Unwilling or unable to believe something \\
Unfriendly & Sarcastic & Ironic & IR & Using words to convey a meaning that is the opposite of its literal meaning \\
Surprised & Scandalized & Scandalized & SC & Shocked or offended by someone else's improper behavior \\
Hurt & Confronted & Confronted & CO & Approached in a critical or threatening way \\
Sorry & Embarrassed & Embarrassed & EM & Worried about what other people will think of you \\
\hline
\end{tabular}

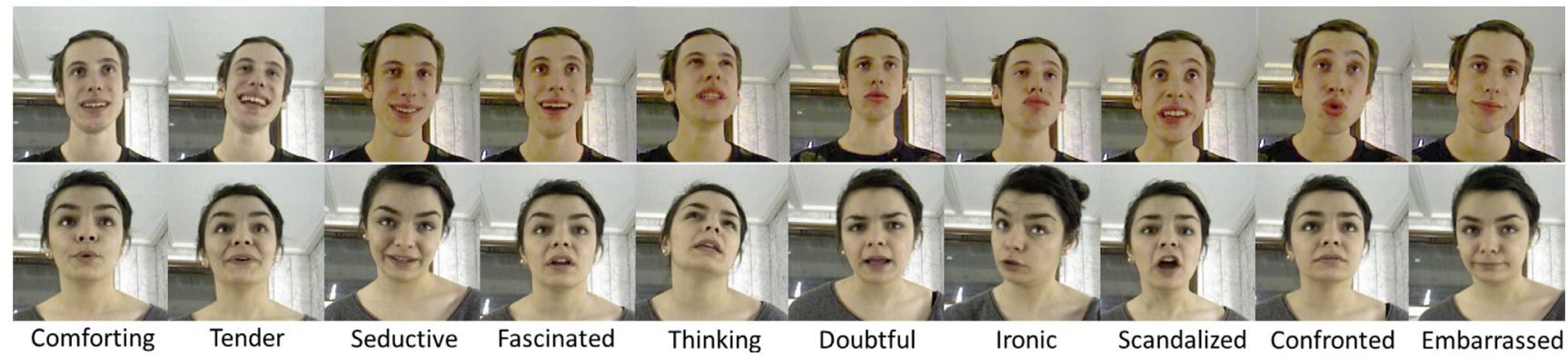

Fig. 1. Examples of the 10 attitudes interpreted by the two actors in our data set.

Massaro, 2003; Cvejic et al., 2010; Sendra et al., 2013), there is no qualitative analysis dedicated to the dynamics of visual prosodic contours of attitudes. We designed and recorded an expressive corpus consisting of attitudes performed by two French speakers. The corpus is designed to include sentences of varied sizes to allow our exploration of discriminating audiovisual features at different structural levels. The data gathered consists both in audio, video and 3D motion capture of the recorded performances. The following section describes the recording process.

\section{Corpus of dramatic attitudes}

We designed and recorded an acted corpus of "pure" social attitudes, i.e. isolated sentences carrying only one attitude over the entire utterance.

Selected text. We extracted the sentences for our database from a French translation of the play "Round dance" by Arthur Schnitzler Schnitzler (2009). The text is represented by 35 sentences (with a distribution of number of syllables spanning between 1 and 21).

Selected attitudes. We selected a subset of 10 attitudes from Baron-Cohen's Mind Reading project (Baron-Cohen, 2003). The source taxonomy proposed by Baron-Cohen comprises a total of 412 attitudes grouped under 24 main categories, each comprising several layers of sub-expressions. The attitude choice was made in collaboration with a theater director, such that the attitudes were compatible with the selected text. Table 2 contains the list of attitudes we decided to analyze for this study. Typical facial displays of these attitudes are illustrated in Fig. 1.

Recordings. The synchronized recording of voice signals and motion was done using the commercial system Faceshift ${ }^{\circledR}$ (http:// www.faceshift.com/) with a short-range Kinect camera and a Lavalier microphone. Faceshift enables the creation of a customized user profile consisting of a 3D face mesh and an expression model characterized by a set of predefined blendshapes that correspond to facial expressions (smile, eye blink, brows up, jaw open etc). Faceshift also outputs estimations of the head motion and gaze direction. The sentences were recorded by two semi-professional actors under the guidance of a theater director. The two actors recorded the 35 sentences uttered first in a neutral, "flat" style, then with each of the selected 10 attitudes. This technique called "exercises in style" is inspired by Queneau (2013) who uses this method of retelling the same story in 99 different styles to train comedians.

The recording session began with an intensive training of the actors, which consisted in fully understanding the interpreted attitudes and developing the ability to dissociate the affective state from the meanings of the sentences. Actors were also instructed to maintain a constant voice modulation, specific for each attitude, throughout uttering the entire set of 35 sentences. The actors performed as if they addressed a person standing in front of them. They did not receive any instructions related to gestural behaviors. A perceptual screening was carried out during the recordings by the theater director and an assistant. If needed, certain utterances were repeated.

Annotation. All utterances were automatically aligned with their phonetic transcription obtained by an automatic text-tospeech phonetizer (Bailly et al., 1991). The linguistic analysis (partof-speech tagging, syllabation), the phonetic annotation and the automatic estimation of melody were further corrected by hand using a speech analysis software (Boersma, 2002). The manual verification of melodic contours represented an extensive effort due to the large amount of data recorded (a total of $3 \mathrm{~h}$ of speech).

\section{Data analysis}

This section presents the analysis of the recorded data: feature extraction, stylization and discriminant analysis.

\subsection{Feature extraction}

There has been a great amount of work on the analysis of features which are found to help in the discrimination between expressive styles. Along with voice pitch (melody), energy, syllable duration and spectrum, we include gestural data: head and eye movements and facial expressions.

Fundamental frequency. As mentioned in the previous section, melody was obtained by automatic phonetic aligning followed by 
manual verification using Praat (Boersma, 2002). Therefore, we obtained reliable $F_{0}$ contours which we further normalized and then converted to semitones.

$f_{0}$ ref $= \begin{cases}210 \mathrm{~Hz}, & \text { if female } \\ 110 \mathrm{~Hz}, & \text { if male }\end{cases}$

$F_{0}[$ tone $]=\frac{240}{\log 2} \log \frac{F_{0}[\mathrm{~Hz}]}{F_{\text {ref }}}$

where $F_{\text {ref }}$ represents the speaker's register $(210 \mathrm{~Hz}$ for the female speaker and $110 \mathrm{~Hz}$ for the male speaker). The resulting $F_{0}$ contours are comparable across speakers.

Rhythm. For rhythm we used a duration model (Campbell, 1992; Bailly and Holm, 2005) where syllable lengthening/ shortening is characterized with a unique z-score model applied to log-durations of all constitutive segments of the syllable. We compute a coefficient of lengthening/shortening $C$ corresponding to the deviation of the syllable duration $\Delta$ relative to an expected duration $\Delta^{\prime}$ :

$C=\frac{\Delta-\Delta^{\prime}}{\Delta^{\prime}}$

$\Delta^{\prime}=(1-r) \cdot \sum_{i} \overline{d_{p_{i}}}+r \cdot D$

where $i$ is the phoneme index within the syllable, $\overline{d_{p_{i}}}$ is the average duration of phoneme $i, D$ is the average syllabic duration ( $=190 \mathrm{~ms}$ here) and $r$ is a weighting factor for isochronicity $(=0.6$ here). We note $C$ as the rhythm coefficient which is computed for every syllable in all sentences in the corpus.

Energy. Energy is extracted at phone-level and computed as mean energy $(\mathrm{dB})$ :

energy $[\mathrm{dB}]=10 * \log 10\left(\frac{\sum_{i=1}^{|y|} y_{i}^{2}}{|y|}\right)$

where $y$ is the acoustic signal segment with the length $|y|$.

Spectrum. The spectrum is extracted using the vocoder STRAIGHT (Kawahara, 2006) which returns the voice spectra, aperiodicities and fundamental frequency. We use 24 mel-cepstral coefficients, from the 2 nd to the 25th (i.e. excluding the energy).

Head and gaze. Head and gaze motion are obtained directly from the processing of the Kinect RGBD data by the

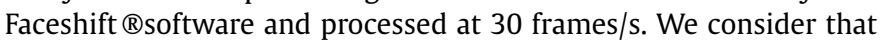
an expressive performance is obtained by adding expressive visual prosodic contours to the trajectories of a "neutral" performance. Since motion has a linear representation, we obtain the visual prosody by simply aligning an expressive performance with its neutral counterpart and computing the difference of the vectors.

Facial expressions. Facial expressions are returned by the Faceshift software as blend shape values. We compute the differential blendshape vectors, to which we apply Non-negative Matrix Factorization (NMF). We split these into two main groups: upperface expressions (8 components) and lower-face expressions (8 components).

\subsection{Feature stylization}

By stylization we mean the extraction of several values at specific locations from the feature trajectories with the main purpose of simplifying the analysis process while maintaining a constant number of characteristics of the original contour for all structural levels whatever the linguistic content. We propose the following stylization methods:
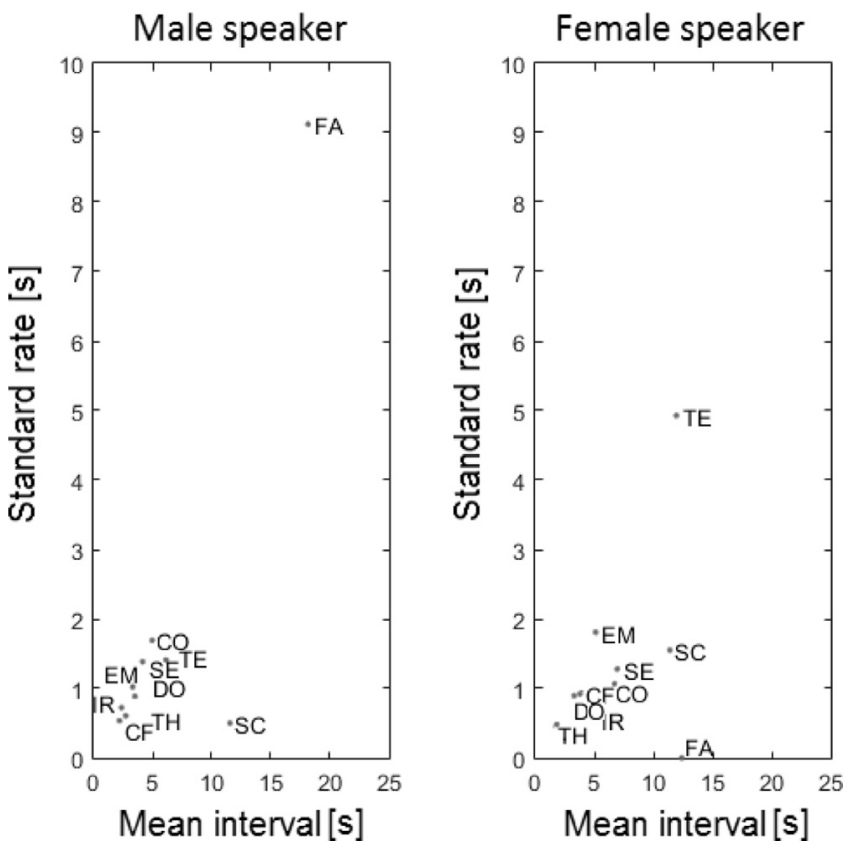

Fig. 2. Mean and standard blinking intervals for the two actors. Note that attitudes such as fascinated, tender and scandalized present higher blinking intervals.

- audio: the audio feature contours are stylized by extracting 3 values: at $20 \%, 50 \%$ and $80 \%$ of the vocalic nucleus of each syllable.

- visual: the visual feature contours are stylized by extracting contour values at $20 \%, 50 \%$ and $80 \%$ of the length of each syllable.

- rhythm: the rhythm is represented by one parameter per syllable: the lengthening/shortening coefficient.

We add virtual syllables to account for the pre- and postphonatory movements for all visual components. As previously observed (Graf etal., 2002), preparatory movements are discriminant to a certain degree for specific emotion categories. We therefore introduce two virtual syllables with a duration of $250 \mathrm{~ms}$ preceding and following each utterance. This duration was chosen because preparatory blinking occurs within $250 \mathrm{~ms}$ of utterance beginning in our dataset. Therefore, stylization of motion is also done for the virtual syllables, by extracting motion contour values at $20 \%, 50 \%$ and $80 \%$ of the duration of each virtual syllable.

\subsection{Blinking interval}

Another prosodic feature we mention in this work is the blinking interval, defined as the time elapsed between consecutive blinks. We extract blinks by thresholding the blendshapes corresponding to eyelid lowering and then we compute the mean and deviation values of the intervals per attitude. Fig. 2 illustrates the blinking strategies presented by the two actors. While this information is useful for attitude characterization, this feature cannot be stylized at syllable level and therefore cannot be used in discriminant analysis at different structural levels.

\subsection{Discriminant analysis}

Discriminant analysis between the 10 attitudes is performed using Fisher classification with 10 -fold cross-validation. Speakerdependent and speaker-independent classification of attitudes were performed at three structural levels for each feature separately, for the concatenation of prosodic features and the concatenation of all audiovisual features: 
Table 3

Dimension and size for all features: F0, rhythm (Rth), energy (Enr), spectrum (Spec), head motion (Head), gaze motion (Gaze), upper-face blendshapes (Up) and lower-face blendshapes (Low).

\begin{tabular}{lllllllll}
\hline & F0 & Rth & Enr & Spec & Head & Gaze & Up & Low \\
\hline Dimension & 1 & 1 & 1 & 24 & 6 & 2 & 8 & 8 \\
Frame size & 1 & - & - & 1 & 1 & 1 & 1 & 1 \\
Syllable size & 3 & 1 & 1 & 3 & 3 & 3 & 3 & 3 \\
Sentence size & 6 & 2 & 2 & 6 & 12 & 12 & 12 & 12 \\
\hline
\end{tabular}

- frame-level: a feature represents data extracted from each stylization point

- syllable-level: a feature represents the concatenation of the frame-level features at each syllable, including virtual syllables for the visual features

- sentence-level: for audio, a feature represents the concatenation of the syllable-level features from the first and last syllables for a given sentence. For visual, we also concatenate the syllablelevel features from the two virtual syllables of that sentence. Note that for sentences composed of one syllable, we perform data duplication to obtain the desired feature dimension (see Table 3).

Results. We analyze our data using F1, a balanced measure between precision and recall. For this, we compute the F1-score, which represents the harmonic mean between precision and recall. We observe that in the case of prosodic features (F0, energy, rhythm, head motion, gaze, facial expressions), the discrimination rate increases as feature granularity increases (see Fig. 3).

Higher scores at sentence-level indicate that order matters: the overall shapes of the features within the sentences have better discrimination power than local feature values. This is especially for F0, head motion and gaze, where the average F1-score is increased by more than $30 \%$ of the scores obtained for the frameand syllable-level. In the case of the concatenated prosodic feature, the gain is smaller. This means that these features already contain enough discriminant information at frame- and syllable-level.

In the case of the spectrum, we observe a decrease in score at sentence-level, showing that the overall shapes at this level does not improve the discrimination of attitudes. The lowest scores for sentence-level features are generally obtained for the speakerindependent classification. F0, head motion, upper and lower-face expressions decrease the most relative to speaker-dependent results, showing that these features manifest different strategies for attitude expression.

Table 4 presents the F1-scores for all features at sentencelevel. On average, high scores are obtained for the F0, head motion and facial expressions, while rhythm and energy show lower discrimination scores. However, attitudes present different score ranges showing that the speakers express audiovisual attitudes using different strategies. For example, Comforting, Fascinated and Ironic show higher scores for the visual features, while Scandalized shows higher audio features. In order to assess the perceptual correlates of these features we carried out two perceptual tests on recordings of the two actors.

\section{Experiments}

\subsection{Perceptual tests}

We carried out two attitude recognition tests using recorded data from the two speakers. The first test used audio and video recordings of the two actors. For the second test, the stimuli were obtained using an animation system, in which the recorded motion is directly mapped to a realistic 3D model of the speaker and the audio signal is represented by the original voice recordings. Both
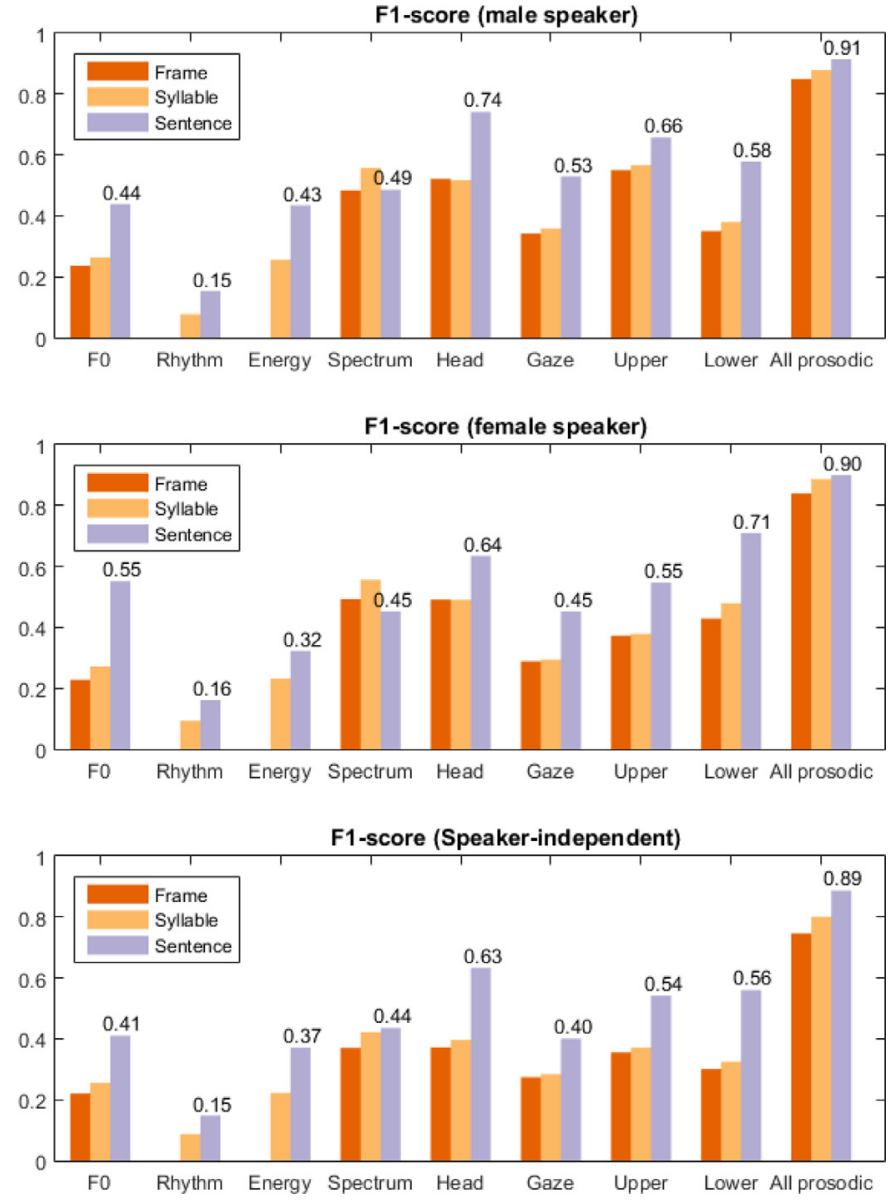

Fig. 3. Average F1-scores obtained for F0, rhythm, energy, spectrum, head motion, gaze motion, upper-face expressions, lower-face expressions and concatenated prosodic features at: frame, syllable and sentence-level. The figures are shown for the male speaker (top), the female speaker (middle) and speaker-independent (bottom). Marked values represent mean F1-scores for sentence-level features.

tests were closed response set with a single choice. The user would play a video and answer the question: "What is the attitude of the actor in this example?" by checking one option from a list of 10 attitudes.

In the first perceptual test, subjects were asked to recognize the attitudes from a total of 40 stimuli, representing audio and video recordings for the two actors. The video modality also included sound. For each actor, the audio modality was presented first. The sentences presented were randomly picked such that any two consecutive stimuli present the different attitudes and different utterances. A total of 20 French native speakers participated in this experience.

In the second perceptual test, each subject is asked to recognize the attitudes of a set of 40 animations representing the two speakers under two modalities: (1) full animation, where all recorded motion is directly retargeted to a 3D model of the actor, (2) headonly animation, where only head motion is retargeted, while facial expressions are fixed. The areas of the 3D head model where expressions are fixed are highlighed by replacing the realistic texture with a matte, gray texture. In the head-only animation modality, the eyes are represented as simple, black holes, thus maintaining the appearance of a mask. These modalities are illustrated in Fig. 5.

The stimuli are presented such that no two consecutive performances contain identical attitudes, sentences or speakers. For one test, random sentences are chosen from a subset of 6 sentences such that each attitude appears twice for each speaker. A total of 
Table 4

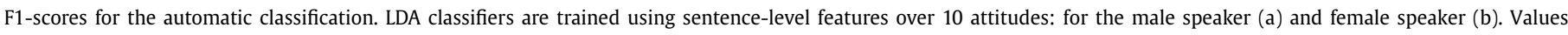
in bold are greater than 0.6 .

\begin{tabular}{|c|c|c|c|c|c|c|c|c|c|c|c|c|c|c|c|c|c|c|c|}
\hline \multicolumn{10}{|c|}{ (a) F1-score for the male speaker } & \multicolumn{10}{|c|}{ (b) F1-score for the female speaker } \\
\hline & F0 & Rth & Enr & Spec & Head & Gaze & Up & Low & All & & F0 & Rth & Enr & Spec & Head & Gaze & Up & Low & All \\
\hline Comforting & 0.24 & 0.23 & 0.48 & 0.33 & 0.76 & 0.62 & 0.64 & 0.58 & 0.90 & Comforting & 0.38 & 0.29 & 0.23 & 0.60 & 0.64 & 0.45 & 0.50 & 0.71 & 0.88 \\
\hline Tender & 0.56 & 0.00 & 0.53 & 0.46 & 0.72 & 0.45 & 0.60 & 0.75 & 0.92 & Tender & 0.68 & 0.08 & 0.47 & 0.49 & 0.36 & 0.38 & 0.36 & 0.85 & 0.89 \\
\hline Seductive & 0.48 & 0.07 & 0.24 & 0.45 & 0.73 & 0.49 & 0.58 & 0.55 & 0.89 & Seductive & 0.47 & 0.10 & 0.26 & 0.31 & 0.76 & 0.34 & 0.64 & 0.69 & 0.89 \\
\hline Fascinated & 0.38 & 0.00 & 0.22 & 0.65 & 0.67 & 0.68 & 0.73 & 0.69 & 0.94 & Fascinated & 0.53 & 0.00 & 0.10 & 0.59 & 0.78 & 0.65 & 0.66 & 0.65 & 0.91 \\
\hline Thinking & 0.62 & 0.37 & 0.50 & 0.27 & 0.82 & 0.43 & 0.71 & 0.39 & 0.94 & Thinking & 0.51 & 0.26 & 0.21 & 0.41 & 0.73 & 0.35 & 0.71 & 0.54 & 0.93 \\
\hline Doubtful & 0.46 & 0.23 & 0.22 & 0.39 & 0.66 & 0.40 & 0.60 & 0.69 & 0.96 & Doubtful & 0.70 & 0.07 & 0.19 & 0.43 & 0.64 & 0.44 & 0.50 & 0.67 & 0.89 \\
\hline Ironic & 0.16 & 0.05 & 0.21 & 0.59 & 0.83 & 0.54 & 0.78 & 0.82 & 0.96 & Ironic & 0.30 & 0.00 & 0.16 & 0.39 & 0.42 & 0.31 & 0.32 & 0.62 & 0.83 \\
\hline Scandalized & 0.68 & 0.22 & 0.87 & 0.39 & 0.81 & 0.49 & 0.60 & 0.43 & 0.90 & Scandalized & 0.65 & 0.30 & 0.92 & 0.78 & 0.57 & 0.26 & 0.48 & 0.60 & 0.97 \\
\hline Confronted & 0.47 & 0.05 & 0.36 & 0.27 & 0.60 & 0.32 & 0.51 & 0.24 & 0.84 & Confronted & 0.68 & 0.04 & 0.41 & 0.68 & 0.63 & 0.50 & 0.51 & 0.90 & 0.99 \\
\hline Embarrassed & 0.43 & 0.21 & 0.68 & 0.87 & 0.82 & 0.73 & 0.73 & 0.83 & 0.99 & Embarrassed & 0.45 & 0.41 & 0.35 & 0.40 & 0.67 & 0.64 & 0.70 & 0.97 & 0.97 \\
\hline Mean & 0.44 & 0.15 & 0.43 & 0.49 & 0.74 & 0.53 & 0.66 & 0.58 & 0.91 & Mean & 0.55 & 0.16 & 0.32 & 0.45 & 0.64 & 0.45 & 0.55 & 0.71 & 0.90 \\
\hline
\end{tabular}
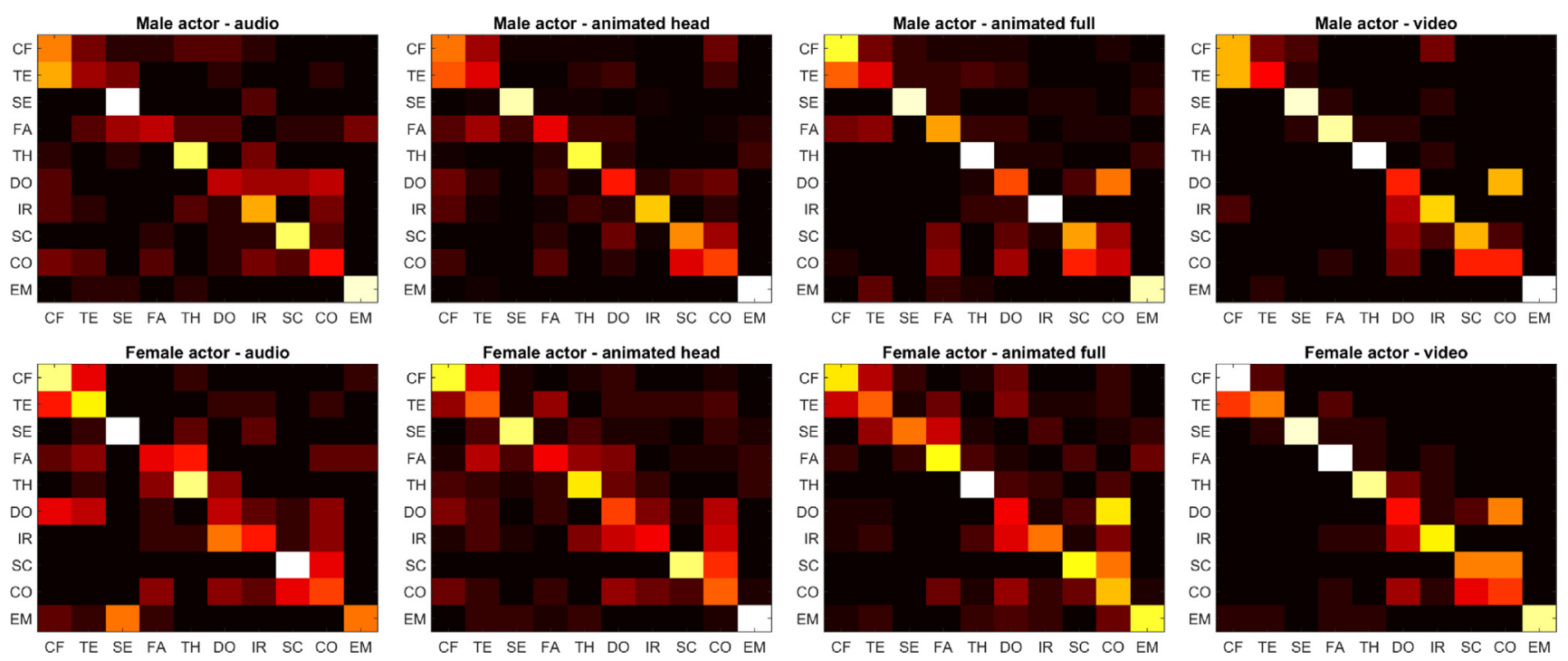

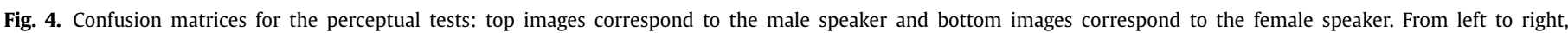

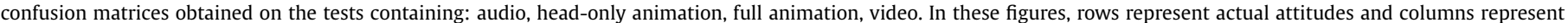
the predictions made by the users. Lighter colors indicate higher values.

Table 5

F1-scores obtained in the perceptual tests, per modality and per actor. Values in bold are greater than 0.6 .

\begin{tabular}{|c|c|c|c|c|c|c|c|c|c|c|c|}
\hline & $\mathrm{CF}$ & $\mathrm{TE}$ & SE & FA & $\mathrm{TH}$ & DO & IR & SC & $\mathrm{CO}$ & EM & Mean \\
\hline \multicolumn{12}{|c|}{ (a) F1-score for the male speaker. } \\
\hline Audio & 0.41 & 0.24 & 0.75 & 0.34 & 0.71 & 0.30 & 0.49 & 0.71 & 0.36 & 0.85 & 0.52 \\
\hline Head & 0.43 & 0.32 & 0.89 & 0.39 & 0.75 & 0.41 & 0.73 & 0.56 & 0.42 & 0.92 & 0.58 \\
\hline Full & 0.60 & 0.33 & 0.86 & 0.52 & 0.82 & 0.43 & 0.90 & 0.52 & 0.25 & 0.83 & 0.61 \\
\hline Video & 0.52 & 0.45 & 0.86 & 0.87 & 0.95 & 0.39 & 0.65 & 0.60 & 0.38 & 0.97 & 0.66 \\
\hline \multicolumn{12}{|c|}{ (b) F1-score for the female speaker. } \\
\hline Audio & 0.54 & 0.48 & 0.70 & 0.29 & 0.60 & 0.21 & 0.36 & 0.71 & 0.34 & 0.52 & 0.48 \\
\hline Head & 0.56 & 0.35 & 0.70 & 0.33 & 0.55 & 0.34 & 0.33 & 0.72 & 0.33 & 0.80 & 0.50 \\
\hline Full & 0.58 & 0.41 & 0.54 & 0.58 & 0.75 & 0.26 & 0.48 & 0.61 & 0.34 & 0.68 & 0.52 \\
\hline Video & 0.77 & 0.59 & 0.92 & 0.82 & 0.80 & 0.36 & 0.70 & 0.53 & 0.33 & 0.89 & 0.67 \\
\hline
\end{tabular}

36 French native speakers participated in this experience. The first online test can be found $\mathrm{at}^{2}$ and the second online test can be found at. $^{3}$

Results. The confusion matrices obtained for the perceptual tests are illustrated in Fig. 4 and the F1-scores are presented in Table 5.

We observe an overall increase in recognition scores as more information is presented to the subjects. The biggest increases are

\footnotetext{
2 http://www.barbulescu.fr/test_audio_video.

3 http://www.barbulescu.fr/test_attitudes.
}

observed between the Audio and Head-only animation for the male actor - especially the case for Ironic - and the Full animation and Video for the female actor - especially the case for the attitudes Comforting, Seductive, Ironic and Embarrassed.

Overall, the best recognized attitudes are Seductive, Thinking, Scandalized and Embarrassed, and lowest are Tender, Doubtful and Confronted, for both speakers. The lowest recognition scores appear because of a high confusion between Tender and Comforting, between Confronted and Scandalized, and an interchangeable confusion between Doubtful and Confronted. This happens because the attitudes in each pair are close in terms of expressive content. 
Table 6

F1-scores obtained for LDA scores vs. perceptual tests, per modality and per actor. Values in bold are greater than 0.6 .

\begin{tabular}{|c|c|c|c|c|c|c|c|c|c|c|c|}
\hline & $\mathrm{CF}$ & $\mathrm{TE}$ & SE & FA & $\mathrm{TH}$ & DO & IR & SC & $\mathrm{CO}$ & EM & Mean \\
\hline \multicolumn{12}{|c|}{ (a) F1-score for the male speaker. } \\
\hline Mod1 & 0.13 & 0.32 & 0.59 & 0.30 & 0.62 & 0.30 & 0.37 & 0.71 & 0.20 & 0.61 & 0.41 \\
\hline Mod2 & 0.43 & 0.32 & 0.70 & 0.39 & 0.75 & 0.41 & 0.73 & 0.56 & 0.38 & 0.77 & 0.54 \\
\hline Mod3 & 0.60 & 0.33 & 0.86 & 0.54 & 0.82 & 0.43 & 0.90 & 0.52 & 0.22 & 0.83 & 0.61 \\
\hline Mod4 & 0.52 & 0.45 & 0.86 & 0.76 & 0.95 & 0.39 & 0.65 & 0.60 & 0.33 & 0.97 & 0.65 \\
\hline \multicolumn{12}{|c|}{ (b) F1-score for the female speaker. } \\
\hline Mod1 & 0.31 & 0.47 & 0.24 & 0.17 & 0.38 & 0.24 & 0.30 & 0.71 & 0.34 & 0.52 & 0.37 \\
\hline Mod2 & 0.52 & 0.31 & 0.67 & 0.33 & 0.29 & 0.34 & 0.28 & 0.67 & 0.40 & 0.67 & 0.45 \\
\hline Mod3 & 0.58 & 0.41 & 0.54 & 0.53 & 0.71 & 0.26 & 0.48 & 0.61 & 0.34 & 0.68 & 0.51 \\
\hline Mod4 & 0.77 & 0.59 & 0.92 & 0.73 & 0.74 & 0.36 & 0.70 & 0.53 & 0.33 & 0.89 & 0.66 \\
\hline
\end{tabular}

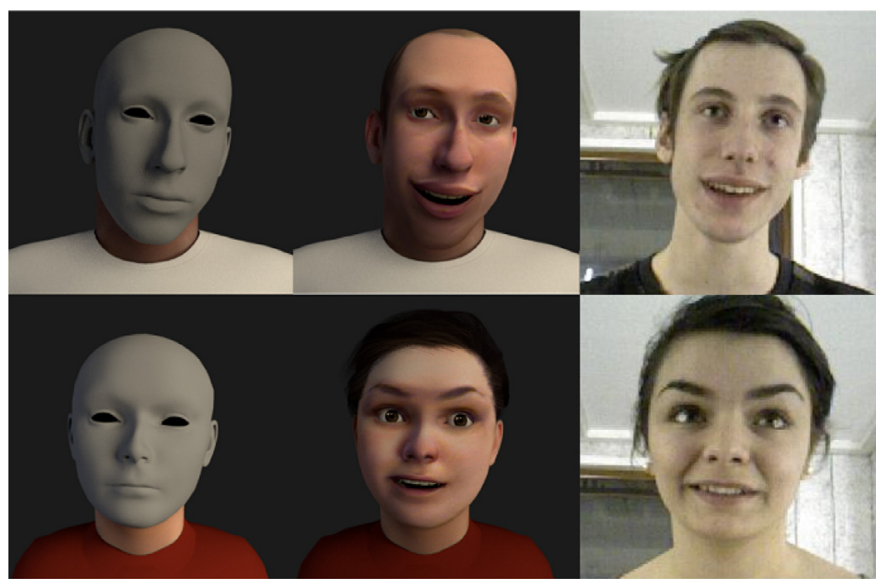

Fig. 5. Corresponding frames for the two speakers extracted from performances of the attitude Comforting, in three modalities: head-only animation, full animation and video.

\subsection{Comparison between subjective and objective scores}

In order to compare the discrimination scores obtained by automatic classification and the perceptual test results, we trained separate LDA classifiers for the two speakers. Data was partitioned into training and testing such that the testing sentences coincide with the ones used in the perceptual test.

After obtaining objective classification results, we are interested in measuring the recognition rates when we consider the classification choices as ground truth data. For this, we compute the confusion matrix where LDA results are predictors and perceptual results are predictions. We test the LDA classifiers on the same sentence that was assessed by the subject of the perceptual test. We are particularly interested in the scores obtained at sentence-level by the concatenation of prosodic features which account for the maximum information that was displayed in the perceptual tests. We therefore define the following feature combinations:

- Mod1 = concatenation of all acoustic prosodic features (F0, energy, rhythm), accounting for the Audio modality in the perceptual test

- Mod2 = concatenation of all acoustic prosodic features and head motion, accounting for the Head-only animation modality in the perceptual test

- Mod3 = concatenation of all prosodic features, accounting for the Full animation modality in the perceptual test

- Mod4 = concatenation of all prosodic features, accounting for the Video modality in the perceptual test

Table 6 presents the F1-scores obtained when we compute the confusion matrices between classification scores for these combi- nations of features and the perceptual scores for their respective modality.

In comparison to the scores presented in Table 5, we observe generally smaller scores for the Audio and Head-only animation modality, and very similar scores for Full animation and Video. In the case of Audio, smaller scores are obtained for attitudes such as Comforting and Seductive, which are performed with specific voice quality ranges. On the other hand Scandalized, which relies on high energy scores, obtains similar scores in all measurements. This shows, that for subtler attitudes, the classification can be improved by fusing a frame-level classifier for segmental features, such as spectrum. The degradation in scores for Head-only animation, can also be attributed to the contribution of acoustic information, since the same attitudes are affected.

We observe a certain variability in terms of speaker-dependent strategies and also in attitude-specific strategies. For example, Fascinated obtains low scores in audio modality and higher scores as more visual information is added, while Scandalized scores high in audio modality and lower as visual information is introduced, due to similarities in visual features with Confronted. For this reason, prosodic features bring different contributions to the perception of dramatic attitudes. This contribution depends both on the attitude itself, but also on the individual strategies of performing.

Interrater agreement. For an in-depth look at the relationship between individual features and perceptual results, we evaluate the agreement between the LDA classification and perceptual raters. For this, we compute Cohen's kappa coefficient (Cohen, 1960) for the confusion matrix obtained by considering LDA results as predictors and subjective results as predictions. The advantage of using this measure is that we are able to compare attitude scoring between raters, by looking at similarities between correctly or incorrectly classified items. The calculation of the kappa coefficient is based on the difference between how much agreement is actually present compared to how much agreement would be expected by chance alone. A perfect agreement yields the value $k=1$, while a chance agreement yields the value $k=0$.

We compute the coefficient on pairs of raters, specifically between each individual rater for the perceptual test and the average LDA rater at frame, syllable and sentence-level. For each pair of raters we test the LDA classifiers on the same sentence that was assessed by the subject of the perceptual test. We perform LDA classification for the following features: F0, concatenated audio, head, gaze, upper-face blendshapes, lower-face blendshapes, concatenated head and audio feature, concatenated audiovisual feature. Fig. 6 presents the coefficients obtained per actor, per feature and per modality.

Our results show that the values of the agreement coefficient increase as granularity increases. This demonstrates a better agreement between our objective scores for sentence-level features and the perceptual scores. For the sentence-level scores, the interrater agreement values obtained range from fair to sub- 

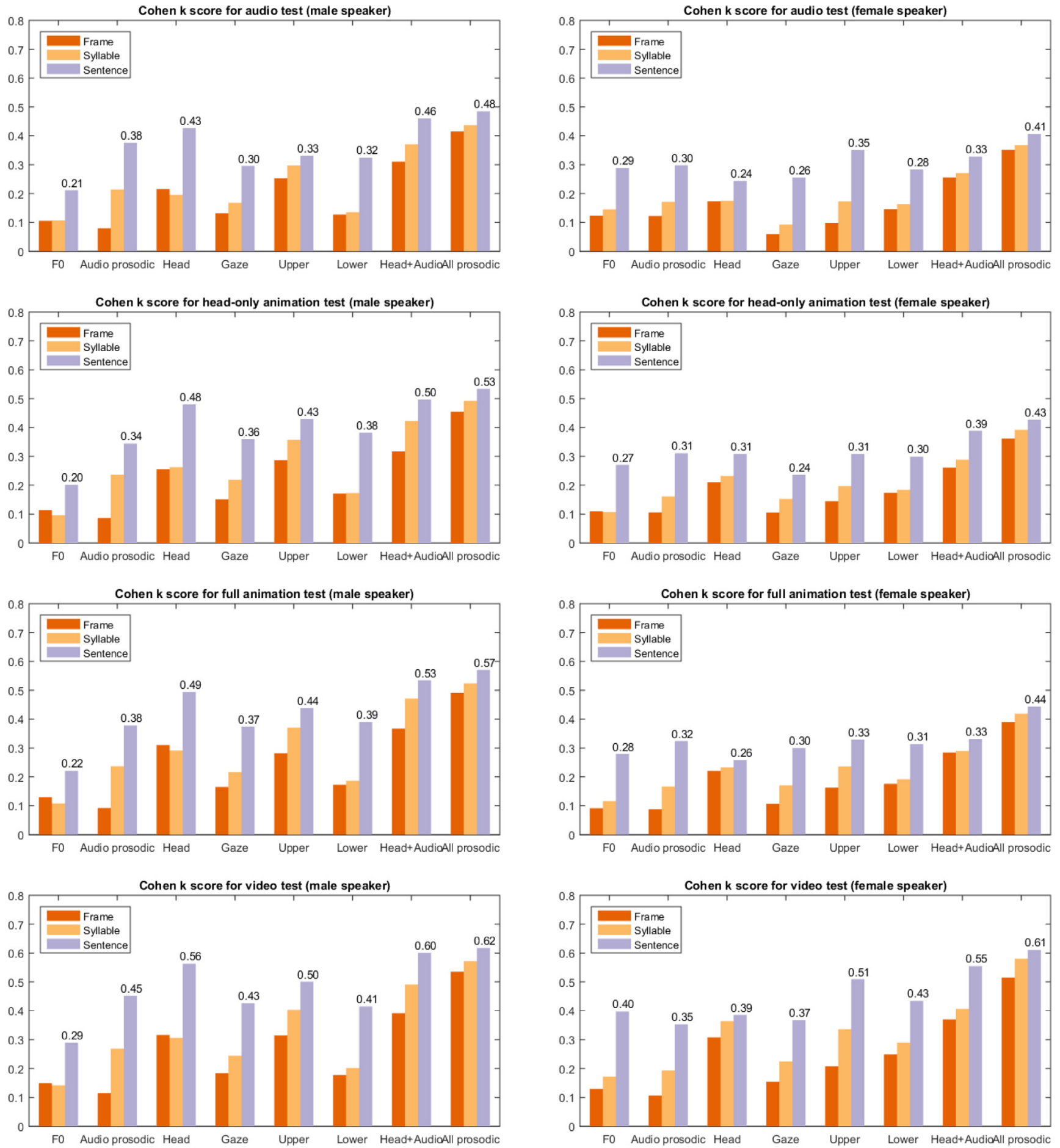

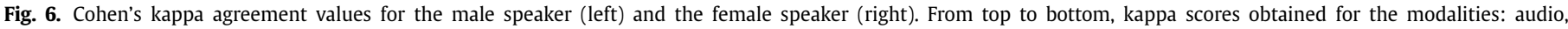
head-only animation, full animation and video.

stantial $(0.21 \leq k \leq 0.62)$, generally with higher agreement for the male actor.

Overall, we observe a higher agreement for head and concatenated head and audio feature for the male actor and higher agreement for F0 for the female actor. Generally, the agreement increases for all features as more information is used in the perceptual tests. A significant increase appears for the female actor for the visual features, as the video modality is used. The lower values for the full animation modality imply that discriminative information - such as subtle expressions and texture - is lost with the us- age of 3D animation. However, the usage of Head-only animation shows a significant increase for head and concatenated head and audio feature for the female actor also.

\section{Conclusion}

In this work, we analyzed audiovisual speech utterances with similar content (sentences) in different styles (dramatic attitudes). We found that the expression of dramatic attitudes is speakerdependent. In a series of experiments, we found that LDA classi- 
fiers trained on speaker-dependent data outperform the classifiers trained on data recorded from both speakers.

We also found that LDA classifiers trained on sentence-level features outperform the classifiers trained on either frame-level or syllable-level features. This means that taking temporal context into account improves the attitude classification performance for prosodic features. The improvement is more significant for F0, head motion and gaze. This is also confirmed by the results of perceptual tests, which show a higher agreement with classification scores obtained for sentence-level features.

In these experiments, we noticed that the speakers use different strategies in the expression of attitudes. The objective evaluation tests show that the male speaker presents higher discrimination rates for the energy, head, gaze and upper-face expressions while the female speaker presents higher scores for the F0 and lowerface expressions. Through perceptual tests, we also proved the effective usage of head motion by the male actor.

Our results show that the studied prosodic features contribute differently to the perceptual discrimination of dramatic attitudes. Future work may include a more in-depth study of the relationship between individual prosodic features and perceptual discrimination of attitudes for a higher number of actors. Animated stimuli can still be valuable as they allow control the amount of visual information provided in a perceptual test.

\section{Acknowledgements}

This work has been supported by the LabEx PERSYVAL-Lab (ANR-11-LABX-0025-01) and the European Research Council advanced grant EXPRESSIVE (ERC-2011-ADG 20110209). We thank Lucie Carta and Grégoire Gouby for their dramatic performances; Estelle Charleroy, Romain Testylier and Laura Paiardini for their art work, and Georges Gagneré for his guidance.

\section{References}

Bailly, G., Barbe, T., Wang, H.-D., 1991. Automatic labeling of large prosodic databases: tools, methodology and links with a text-to-speech system. In: The ESCA Workshop on Speech Synthesis, pp. 77-86.

Bailly, G., Holm, B., 2005. SFC: a trainable prosodic model. Speech Commun. 46 (3), 348-364.

Barbulescu, A., Bailly, G., Ronfard, R., Pouget, M., 2015. Audiovisual generation of social attitudes from neutral stimuli. Facial Analysis, Animation and Auditory-Visual Speech Processing.

Baron-Cohen, S., 2003. Mind Reading: The Interactive Guide to Emotions. Jessica Kingsley Publishers.

Boersma, P., 2002. PRAAT, a system for doing phonetics by computer. Glot Int.l 5 (9/10), 341-345.

Bolinger, D., 1989. Intonation and Its Uses: Melody in Grammar and Discourse. Stanford University Press.

Busso, C., Bulut, M., Lee, C.-C., Kazemzadeh, A., Mower, E., Kim, S., Chang, J.N., Lee, S., Narayanan, S.S., 2008. IEMOCAP: interactive emotional dyadic motion capture database. Lang. Resour. Eval. 42 (4), 335-359.

Busso, C., Deng, Z., Grimm, M., Neumann, U., Narayanan, S., 2007. Rigid head motion in expressive speech animation: analysis and synthesis. Audio, Speech, Lang. Process., IEEE Trans. 15 (3), 1075-1086.

Busso, C., Deng, Z., Yildirim, S., Bulut, M., Lee, C.M., Kazemzadeh, A., Lee, S., Neumann, U., Narayanan, S., 2004. Analysis of emotion recognition using facial expressions, speech and multimodal information. In: Proceedings of the 6th International Conference on Multimodal Interfaces. ACM, pp. 205-211.

Campbell, W.N., 1992. Syllable-based segmental duration. Talking Machines: Theories, Models, and Designs 211-224.

Cohen, J., 1960. A coefficient of agreement for nominal scale. Educ. Psychol. Measurement 20, 37-46.

Cowie, R., Douglas-Cowie, E., Cox, C., 2005. Beyond emotion archetypes: databases for emotion modelling using neural networks. Neural Netw. 18 (4), 371-388.

Cvejic, E., Kim, J., Davis, C., Gibert, G., 2010. Prosody for the eyes: quantifying visual prosody using guided principal component analysis. In: Interspeech, pp. $1433-1436$.

Davis, C., Kim, J., Aubanel, V., Zelic, G., Mahajan, Y., 2015. The stability of mouth movements for multiple talkers over multiple sessions. In: Proceedings of the 2015 FAAVSP.
De Moraes, J.A., Rilliard, A., de Oliveira Mota, B.A., Shochi, T., 2010. Multimodal perception and production of attitudinal meaning in brazilian portuguese. Proc. Speech Prosody, Paper 340.

Ekman, P., Friesen, W.V., 1971. Constants across cultures in the face and emotion. J. Pers. Soc. Psychol. 17 (2), 124

Fanelli, G., Gall, J., Romsdorfer, H., Weise, T., Van Gool, L., 2010. A 3d audio-visual corpus of affective communication. Multimedia, IEEE Trans. 12 (6), 591-598.

Fónagy, I., Bérard, E., Fónagy, J., 1983. Clichés mélodiques. Folia Linguistica 17 (1-4), $153-186$.

Graf, H.P., Cosatto, E., Strom, V., Huang, F.J., 2002. Visual prosody: facial movements accompanying speech. In: Automatic Face and Gesture Recognition, 2002. Proceedings. Fifth IEEE International Conference on. IEEE, pp. 396-401.

Granström, B., House, D., Swerts, M., 2002. Multimodal feedback cues in humanmachine interactions. In: Speech Prosody 2002, International Conference.

Hönemann, A., Mixdorff, H., Rilliard, A., 2014. Social attitudes - recordings and evaluation of an audio-visual corpus in German. Forum Acusticum.

Hnemann, A., Mixdorff, H., Rilliard, A., 2015. Classification of auditory-visual attitudes in German. In: International Conference on Auditory-Visual Speech Processing.

Iriondo, I., Planet, S., Socoró, J.-C., Alías, F., 2007. Objective and Subjective Evaluation of an Expressive Speech Corpus. In: Advances in Nonlinear Speech Processing. Springer, pp. 86-94.

Iriondo, I., Planet, S., Socoró, J.-C., Martínez, E., Alías, F., Monzo, C., 2009. Automatic refinement of an expressive speech corpus assembling subjective perception and automatic classification. Speech Commun. 51 (9), 744-758.

Kaulard, K., Cunningham, D.W., Bülthoff, H.H., Wallraven, C., 2012. The MPI facial expression database a validated database of emotional and conversational facial expressions. PLoS One 7 (3), e32321.

Kawahara, H., 2006. STRAIGHT, exploitation of the other aspect of vocoder: perceptually isomorphic decomposition of speech sounds. Acoust. Sci. Technol. 27 (6) 349-353.

Lucey, P., Cohn, J.F., Kanade, T., Saragih, J., Ambadar, Z., Matthews, I., 2010. The extended Cohn-Kanade dataset (ck+): acomplete dataset for action unit and emotion-specified expression. In: Computer Vision and Pattern Recognition Workshops (CVPRW), 2010 IEEE Computer Society Conference on. IEEE, pp. 94-101.

Mac, D.-K., Castelli, E., Aubergé, V., 2012. Modeling the prosody of Vietnamese attitudes for expressive speech synthesis. In: SLTU, pp. 114-118.

Mahmoud, M., Baltrušaitis, T., Robinson, P., Riek, L.D., 2011. 3D corpus of spontaneous complex mental states. In: Affective Computing and Intelligent Interaction. Springer, pp. 205-214.

Mixdorff, H., Hönemann, A., Rilliard, A., 2015. Acoustic-prosodic analysis of attitudinal expressions in german. In: Proceedings of Interspeech 2015.

Monzo, C., Alías, F., Iriondo, I., Gonzalvo, X., Planet, S., 2007. Discriminating expressive speech styles by voice quality parameterization. Proceedings of ICPhS.

Morlec, Y., Bailly, G., Aubergé, V., 2001. Generating prosodic attitudes in french: data, model and evaluation. Speech Commun. 33 (4), 357-371.

Ouni, S., Colotte, V., Dahmani, S., Azzi, S., 2016. Acoustic and visual analysis of expressive speech: acase study of French acted speech. In: Interspeech 2016, 2016 pp. 580-584.

Pelachaud, C., Badler, N.I., Steedman, M., 1996. Generating facial expressions for speech. Cogn. Sci. 20 (1), 1-46.

Queneau, R. 2013. Exercises in Style. New Directions Publishing.

Savran, A., Alyüz, N., Dibeklioğlu, H., Çeliktutan, O., Gökberk, B., Sankur, B. Akarun, L., 2008. Bosphorus database for 3D face analysis. In: Biometrics and Identity Management. Springer, pp. 47-56.

Scherer, K.R., 1986. Vocal affect expression: a review and a model for future research. Psychol. Bull. 99 (2), 143.

Scherer, K.R., Wallbott, H.G., 1994. Evidence for universality and cultural variation of differential emotion response patterning. J. Pers. Soc. Psychol. 66 (2), 310.

Scherer, U., Helfrich, H., Scherer, K., 1979. Paralinguistic behaviour: internal push or external pull? In: Language: Social Psychological Perspectives: Selected Papers from the First International Conference on Social Psychology and Language held at the University of Bristol, England, p. 279.

Schnitzler, A., 2009. Round Dance. Oxford University Press.

Sendra, V.C., Kaland, C., Swerts, M., Prieto, P., 2013. Perceiving incredulity: the role of intonation and facial gestures. J. Pragmatics 47 (1), 1-13.

Srinivasan, R.J., Massaro, D.W., 2003. Perceiving prosody from the face and voice: distinguishing statements from echoic questions in english. Lang. Speech 46 (1), $1-22$.

Swerts, M., Krahmer, E., 2010. Visual prosody of newsreaders: effects of information structure, emotional content and intended audience on facial expressions. J. Phon. 38 (2), 197-206.

Vandeventer, J., Aubrey, A.J., Rosin, P.L., Marshall, D., 2015. 4d Cardiff conversation database $(4 \mathrm{~d} c \mathrm{cdb})$ : a $4 \mathrm{~d}$ database of natural, dyadic conversations. In: International Conference on Auditory-Visual Speech Processing.

Zeng, Z., Pantic, M., Roisman, G.I., Huang, T.S., 2009. A survey of affect recognition methods: audio, visual, and spontaneous expressions. IEEE Trans. Pattern Anal. Mach. Intell. 31 (1), 39-58. 